Reprod. Nutr. Dévelop., 1983, 23 (2 B), 385-387.

\title{
Effets de modifications aiguës de l'insulinémie endogène sur la distribution subcellulaire des récepteurs de l'insuline dans le foie de rat
}

\author{
Soledad LOPEZ, B. DESBUQUOIS
}

Unité 30 INSERM, Hôpital des Enfants Malades, 149, rue de Sèvres, 75730 Paris Cedex 15.

Summary. The effects of acute changes in endogenous insulinemia on the subcellular distribution of insulin receptors in rat liver.

The effects of acute changes in endogenous insulinemia on the number and subcellular distribution of insulin receptors in rat liver have been studied. After injecting stimulants or inhibitors of insulin secretion, we examined various subcellular fractions from male rats $(200 \mathrm{~g})$ for their ability to specifically bind ${ }^{125}$-insulin. Glucose $(300 \mathrm{mg})$ elevated plasma insulin 4 to 6-fold and increased the number of insulin receptors in Golgi fractions by $50 \%$; it decreased the number of receptors in plasma membrane by $20 \%$. These changes were maximal by 5-15 min and reversible in one hour. Arginine $(200 \mathrm{mg}$ ), glucagon $(75 \mu \mathrm{g})$ and tolbutamide $(10 \mathrm{mg})$, which elevated insulinemia to the same extent as glucose, also increased insulin binding to Golgi fractions. Conversely, somatostatin $(40 \mu \mathrm{g})$ and diazoxide $(10 \mathrm{mg})$, which lowered plasma insulin 3 to 4 -fold, decreased insulin binding to Golgi fractions by $20-25 \%$. In all cases, the total number of receptors measured on a crude particulate fraction was not affected. Thus, acute changes in endogenous insulinemia caused a redistribution of insulin receptors in hepatocytes. It is suggested that this redistribution resulted from changes in the internalization rate of the receptors, which itself was a function of the degree of receptor occupancy.

\section{Introduction.}

S'il est généralement admis que l'insuline exerce une régulation négative sur le nombre de ses récepteurs dans la membrane cellulaire, peu d'informations existent sur les modifications des récepteurs intracellulaires. En particulier, on ignore si la diminution des récepteurs dans les cellules cibles après l'exposition à I'hormone reflète une diminution du nombre total des récepteurs ou simplement leur redistribution à l'intérieur de la cellule.

Nous avons récemment montré que, chez le rat, une injection unique d'insuline diminuait le nombre des récepteurs dans la membrane plasmique et, réciproquement, l'augmentait dans les fractions golgiennes; le nombre total des récepteurs n'était pas affecté. Cette observation nous a conduits à proposer que 
l'occupation des récepteurs par leur ligand provoquait leur translocation de la surface vers l'intérieur de l'hépatocyte (Desbuquois et al., 1979, 1982).

Le but de ce travail a été d'étudier les effets de modifications aiguës de l'insulinémie endogène, de caractère plus physiologique, sur le nombre et la distribution subcellulaire des récepteurs de l'insuline dans le foie de rat.

\section{Matériel et méthodes.}

Des rats mâles de $200 \mathrm{~g}$ ont reçu divers stimulants ou inhibiteurs de la sécrétion d'insuline. Les premiers ont inclus le glucose $(300 \mathrm{mg})$, l'arginine $(200 \mathrm{mg})$, le glucagon $(75 \mu \mathrm{g})$ et la tolbutamide $(10 \mathrm{mg})$; les seconds, la somatostatine $(40 \mu \mathrm{g})$ et le diazoxide $(10 \mathrm{mg})$. A différents temps les animaux ont été sacrifiés ; le foie a été prélevé, homogénéisé et fractionné. Une fraction particulaire totale, des membranes plasmiques (Neville, 1968) et des fractions golgiennes (Ehrenreich et al., 1973) ont été préparées. Ces fractions ont été examinées, après solubilisation par le Triton X-100, pour leur capacité à lier spécifiquement l'insuline${ }^{125}$. La concentration de l'insuline dans le plasma et dans les extraits des fractions subcellulaires a été déterminée par dosage radioimmunologique.

\section{Résultats.}

1) L'injection d'une solution de glucose, qui augmente de 4 à 6 fois l'insulinémie, élève de $50 \%$ la liaison de l'insuline aux fractions golgiennes; réciproquement, elle la diminue de $20 \%$ dans les membranes plasmiques. Ces modifications, légèrement différées par rapport au pic d'insulinémie, sont maximales entre 5 et $15 \mathrm{~min}$; elles disparaissent en une heure.

2) Les modifications de la liaison de l'insuline aux fractions golgiennes chez les animaux ayant reçu une injection de glucose sont indépendantes de la concentration de l'insuline dans le milieu d'incubation. Cette observation indique une modification du nombre des récepteurs et non pas de leur affinité.

3) L'augmentation de la liaison de l'insuline aux fractions golgiennes s'accompagne d'une augmentation marquée ( 4 à 6 fois) de leur teneur en insuline immunoréactive. Cette observation suggère que les récepteurs dans les fractions golgiennes des animaux traités par le glucose sont présents sous forme de complexes insuline-récepteur.

4) L'injection d'arginine, de glucagon et de tolbutamide, qui élèvent l'insulinémie aux mêmes taux que le glucose, provoquent aussi une augmentation de $50 \%$ de la liaison de l'insuline aux fractions golgiennes.

5) Contrairement aux agents précédents, la somatostatine et le diazoxide, qui abaissent d'environ 4 fois l'insulinémie à $15 \mathrm{~min}$, diminuent dans le même temps de 20 à $25 \%$ la liaison de l'insuline aux fractions golgiennes.

6) Dans aucune des situations précédentes la liaison à la fraction particulaire totale n'est modifiée ; cette observation indique que le nombre total des récepteurs est inchangé. 


\section{Discussion et conclusions.}

Cette étude montre qu'une élévation aiguë de l'insulinémie endogène chez le rat élève le nombre des récepteurs de l'insuline dans les fractions golgiennes et le diminue dans les membranes plasmiques; le nombre total des récepteurs est inchangé. Ces altérations sont rapides et réversibles ; elles coïncident approximativement avec le pic d'hyperinsulinémie. Réciproquement, une diminution aiguë de l'insulinémie diminue le nombre des récepteurs dans les fractions golgiennes. Nous suggérons que les modifications de la distribution subcellulaire des récepteurs résultent de changements dans leur degré d'internalisation, lui-même fonction de leur degré d'occupation par l'hormone.

\footnotetext{
ge Réunion du groupe, Développement I.N.R.A.,
} Tours, 12-13 mai 1982.

\section{Références}

DESBUQUOIS B., WILLEPUT J., HUET DE FROBERVILLE A., 1979. Receptor-mediated internalization of insulin in intact rat liver. FEBS Lett., 106, 338-344.

DESBUQUOIS B., LOPEZ S., BURLET H., 1982. Ligand-induced translocation of insulin receptors in intact rat liver. J. biol. Chem., 257, 10852-10860.

EHRENREICH J. H., BERGERON J. J. M., SIEKEVITZ P., PALADE G. E., 1973. Golgi fractions prepared from rat liver homogenates. I. Isolation procedure and morphological characterization. J. Cell Biol., 59, 45-72.

NEVILLE D. M., 1968. Isolation of an organ specific protein antigen from cell surface membrane of rat liver. Biochim. biophys. Acta, 154, 540-552. 